\section{Kidney \\ Blood Pressure Research}

\title{
Cystatin C is Associated With Plaque Phenotype and Plaque Burden
}

\author{
Yufeng Wen ${ }^{a}$ Dan Xia ${ }^{a}$ Yanchun Wanga,b Huiping Zhang ${ }^{c}$ Haibo Lia Gholam \\ Ali $^{d}$ Yongqing Gao Jian Lif ${ }^{\mathrm{e}}$ Wenjie Sun ${ }^{\mathrm{e}}{ }^{\mathrm{f}}$ Linlin Li ${ }^{g}$
}

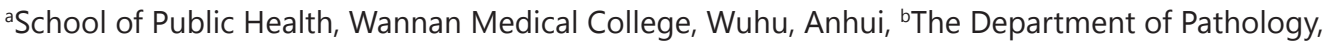
School of Medicine, The Fourth Affiliated Hospital, Zhejiang University, Yiwu, Zhejiang, 'Department of Medical Ultrasonics, Ma'anshan People's Hospital, Ma'anshan, Anhui, China; 'School of Medicine, Tulane University, New Orleans, LA, USA; eSchool of Food Science, Guangdong Pharmaceutical Univer-

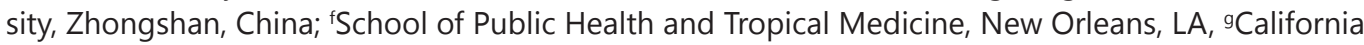
Department of Public Health, Sacramento, California, USA

\section{Key Words}

Cystatin C • Plaque phenotype $\cdot$ Plaque burden $•$ Renal function

\begin{abstract}
Background/Aims: The relationship between carotid artery plaque burden, phenotype and serum cystatin $C$ at normal and impaired renal function is still unclear. Methods: Demographic characteristics, carotid ultrasonography and other relevant information of 1,477 patients were collected. The association of carotid artery plaque burden, plaque phenotype with serum cystatin $C$ was evaluated by strategy analysis based on renal function. Results: Serum cystatin C $(O R=2.05,95 \% \mathrm{CI}: 1.83-2.29, P<.01)$ was a risk factor of stable plaque among patients with normal glomerular filtration rate. However, in the patients with mild impaired renal function, serum cystatin $C$ was not only a risk factor for stable plaque $(O R=1.60,95 \% C I$ : $1.43-1.78$, $P<.001)$ but also was a risk factor for unstable plaque (OR=1.21, 95\%CI: $1.10-1.32, P<.001)$. The smoothing function curve and a three-piecewise linear regression revealed that a nonlinear relationship was observed between serum cystatin $C$ and plaque burden. When serum cystatin $\mathrm{C}$ was in the range of $0.75-1.30$ ( $\mathrm{mg} / \mathrm{L}$ ), the plaque burden tended to increase. Conclusion: In normal renal function, serum cystatin $C$ may confer stability of plaques. In mildly impaired renal function, serum cystatin $C$ is a risk predictor of plaques. In normal renal function circumstances, serum cystatin $C$ may benefit to the stability of plaques. In mild impaired renal function circumstances, serum cystatin $C$ are a risk predictors of plaques.
\end{abstract}




\section{Kidney Blood Pressure Research}

Kidney Blood Press Res 2016;41:197-207

DOI: $10.1159 / 000443422$

Published online: March 25, 2016

(C) 2016 The Author(s). Published by S. Karger AG, Base

www.karger.com/kbr

Wen/Xia/Wang/Zhang/Li/Ali/Gao/Li/Sun/Li: A Clinic Based Study

\section{Introduction}

Atherosclerosis (AS) is a disease characterized by chronic inflammation in large- and medium-sized arteries. Carotid atherosclerosis (CAS) is not only an early manifestation of systemic AS but also the early phase reaction of cardiovascular diseases (CVD) [1]. Carotid artery plaque burden and plaque phenotype are two main indicators of generalized AS [2, $3]$, and relate to greater risks of cardiovascular and all-cause mortality [4, 5]. Presence of atherosclerotic plaques in the internal carotid artery is associated with an increased risk of incident stroke even in asymptomatic adults aged 65 years or older [6].

A large number of prospective studies suggest that metabolic syndrome, smoking and alcohol drinking are major risk factors of CAS [7-9], and increase CVD morbidity and mortality in the general population $[10,11]$. Recent studies found that in addition to the above risk factors, the elevated serum cystatin C (cys C) was an independent predictor of adverse cardiovascular outcomes among patients without established chronic kidney disease [12]. Cys $\mathrm{C}$, which is produced by all nucleated cells, belongs tothe cystatin superfamily of endogenous cysteine protease inhibitors and could inhibit the protease of cathepsin. Cys C has recently been suggested as a more reliable marker of renal function compared to serum creatinine and blood urea nitrogen $[13,14]$. Our previous studies found that Cys C, an alternative measure of renal function, had a stronger impact on carotid thickening and plaque formation than other measures of renal function $[15,16]$. CAS is an inflammatory disease characterizing by extracellular matrix (ECM) remodeling (formation and degradation) in vascular wall. The dynamic balance of formation and degradation in ECM plays a key role in the development and progression of CAS [17]. Cys C can inhibit the activity of cathepsin and the degradation of ECM in the progression of atherosclerosis [18]. Unfortunately, in recent years, findings in terms of the effect of Cys C onCVD remained controversial. Muntner et al. found that a graded association existed between higher serum Cys $\mathrm{C}$ and increased CVD prevalence in patients without established chronic kidney disease [19]. However, Doganer et al found that lower Cys C levels were associated with increased severity of coronary artery disease [20]. Furthermore, previous studies failed to take into accountthe effect of renal function on Cys $\mathrm{C}$ levels. Therefore the aims of this study were to examine the relationship between Cys $\mathrm{C}$ and carotid artery disease stratified by varying levels of renal function, and thereby provide informative guidance in preventing severe cardiovascular and cerebrovascular events.

\section{Materials and Methods}

\section{Study population}

A total of 1,477 patients have been recruited in the Department of Medical Ultrasonics in Ma'anshan People's Hospital, China from December 2013 to June 2014. The inclusion criteria were: (a) age from 40 to 75 years; (b) without severe cardiovascular diseases; (c) creatinine derived glomerular filtration rate (GFR) $\geq 60 \mathrm{ml} / \mathrm{min} / 1.73 \mathrm{~m}^{2}$; (d) without stroke history; (e) without carotid intervention history; (f) without severe infection and (g) tumor history. Informed consents were obtained from all participants (The medical ethics committee of Ma'anshan People's Hospital, No. 2014002). The sample was divided into two groups based on patients' renal function levels: normal renal function $(\mathrm{n}=900)$ and mild impaired renal function $(n=577)$, while three subgroups (control group, stable plaque group, and unstable plaque group) were categorized within each group according to the results of carotid ultrasonography analysis. Thus among patients who had normal renal function, 381 were in the control group, and 242 patients and 277 patients were in the stable plaque and unstable plaque groups, respectively. Among patients who were classified as mild impaired renal function, the subsamples assigned in the control/stable plaque/unstable plaque groups were 279,119 , and 179 , respectively. 


\section{Kidney Blood Pressure Research}

Wen/Xia/Wang/Zhang/Li/Ali/Gao/Li/Sun/Li: A Clinic Based Study

\section{Variables}

The questionnaire was designed by clinical experts and experienced epidemiologists and covered multiple components, including demographic characteristics (age and sex), behaviors characteristics and body examination., Behavior characteristics include smoking (regular smoking: smoking $\geq 1$ cigarette/day, time of smoking duration $\geq 6$ months; occasional smoking: smoking $\geq 4$ times/week, but $<1$ cigarette/day or never smoking), drinking (currently drinkers: $56^{\circ}$ alcohol liquor $\geq 50 \mathrm{ml} /$ day, $>5$ times/week; occasional drinkers: $56^{\circ}$ alcohol liquor $<50 \mathrm{ml} /$ day, $\leq 4$ times/week or never drinker), and physical exercise (regularly exercise: exercise $\geq 30 \mathrm{~min} /$ time, 3-7 times/week; occasional: exercise $<30 \mathrm{~min} /$ time, $<3$ times/week or never). The body examination includes height, weight, body mass index (BMI, calculated as: weight (kg)/ square of height $\left(\mathrm{m}^{2}\right)$ ). blood pressure, fasting blood glucose. Hypertension was defined as systolic blood pressure (SBP) $\geq 140 \mathrm{~mm} \mathrm{Hg}$ and/or diastolic blood pressure (DBP) $\geq 90 \mathrm{~mm} \mathrm{Hg}$ and/or treated hypertension. Patients with fasting blood glucose $>7.1 \mathrm{mmol} / \mathrm{L}$ or treated diabetes were classified as diabetes.

\section{Laboratory assay}

For each patient who had fasted for 12 hours, a 5-7 ml venous blood was draw and then centrifuged and stored at $-70^{\circ} \mathrm{C}$ until assayed. Serum Cys $\mathrm{C}$ was determined by immunoturbidimetric assay (Inter-assay and intra-assay coefficients of variation were $<3.6 \%$ and $<2.7 \%$, respectively). Fasting blood glucose was determined by glucose oxidase method, serum total cholesterol (TC) and triglyceride (TG) were determined by CHOD-PAP, high-density lipoprotein (HDL) was determined by phosphotungstic acid-magnesium precipitation, low-density lipoprotein(LDL) was determined by selective removal method. The serum creatinine (Cr) was analyzed using the Jaffe method, and traceable to the International Standardization with isotope dilution mass spectometry (IDMS). Estimated glomerular filtration rate (GFR) was calculated by Modification of diet in renal disease (MDRD) formula as follows: eGFR (ml/ min/ $1.73 \mathrm{~m}^{2}$ ) =175× [serum creatinine $(\mathrm{mg} / \mathrm{dl}) / 88.41(\mu \mathrm{mol} / \mathrm{L})]^{-1.154} \times$ Age (years) $)^{-0.203}(\times 0.742$ if female) [21]. All biochemistry assays were performed with the automatic biochemical analyzer (DPP-800, Roche, Germany) by professional laboratory technician in hospital.

\section{Carotid Ultrasonography}

The near and far walls of the common carotid, the internal carotid, bifurcation, and internal carotid artery on both sides were scanned to examine the presence (yes/no) of atherosclerotic plaques using 5-13 MHz linear-array probes (Aloka-a7 and Aloka-a10, Japan; Philips-IU22, America) [5].

Patients were examined in the supine position with the head turned $45^{\circ}$ from the side being scanned. Each carotid system was scanned in anterior oblique, lateral, and posterior oblique planes. Near and far walls of the common carotid artery began just above the clavicle and was moved cephalad through the bifurcation and along both the internal and external branches as far distally as possible. The sonographer consistently aimed for the clearest image of the far wall of the carotid artery so as to be certain that the chord of greatest length was achieved, and therefore that the image represented the true diameter of the vessel. The carotid intima media thickness (IMT) protocol consisted of scanning the carotid arteries longitudinally in the 3 segments, using the lateral extent of each carotid segment as defined relative to the tip of the flow divider, which is normally the most clearly defined anatomic reference in the proximity of the carotid bifurcation. The carotid segments were defined as follows: (1) near and far wall of the segment extending from 10 to $20 \mathrm{~mm}$ proximal to the tip of the flow divider into the common carotid artery; (2) near and far wall of the carotid bifurcation beginning at the tip of the flow divider and extending $10 \mathrm{~mm}$ proximal to the flow divider tip; and (3) near and far wall of the proximal $10 \mathrm{~mm}$ of the internal carotid artery. Fine transducer angulations were used to clearly display both the blood-intima and media-adventitia boundaries on both the near and far walls of the artery, and the transducer was moved toward the mandible until the lumen area increased with the appearance of the carotid bifurcation, and finally the internal carotid artery was visualized. The focus was positioned at the $40-\mathrm{mm}$ depth of the near or the far wall depending on the optimized image during scanning. In the case of plaque presence, carotid IMT was measured outside the portion of plaque.

Carotid plaque, defined as was defined as local thickening of the IMT of 50\% compared to the surrounding vessel wall, an IMT $\geq 1.5 \mathrm{~mm}$, or local thickening $\geq 0.5 \mathrm{~mm}$ (as evidenced by protrusion into the lumen and/or localized roughness with increased echogenicity) [3], was classified by surface characteristics, 


\section{Kidney \\ Blood Pressure Research}

Kidney Blood Press Res 2016;41:197-207

\begin{tabular}{|l|l|}
\hline DOI: 10.1159/000443422 & ( 2016 The Author(s). Published by S. Karger AG, Basel
\end{tabular}

Published online: March 25, 2016

www.karger.com $/ \mathrm{kb}$

echogenicity, and texture. The carotid atherosclerotic plaques were classified as stable or unstable. Stable plaques were required to have all of the following features: (a) smooth and regular surface; (b) homogeneity and uniform echo geneity or dominant echo geneity with small areas of echo lucency. Unstable plaques were required to have one or more of the following features: (a) ulceration (isolated crater $>1 \mathrm{~mm}$ with proximal and distal lipping); (b) irregular surface; (c) presence of mobile thrombi on the plaque surface or intraluminal thrombus; (d) hypoechoic plaques ; (f) heterogeneity with substantial intraplaque echolucent areas [22]. Carotid plaque area was determined as the sum cross-sectional area of all carotid plaques (IMT $\geq 1.5$ $\mathrm{mm}$ ) in all carotid vessels (common, internal, and bifurcations) bilaterally. Each longitudinal plaque image was corroborated against axial images to assure accurate imaging (to reduce overestimation or underestimation of plaque size) [3]. Plaque score was calculated according to the sum of the largest thickness of each individual plaque [23].

\section{Statistical analysis}

Descriptive statistics were showed as mean \pm SD for continuous variables and frequency (\%) for categorical variables. Differences in baseline characteristics between levels of renal function were compared using ANOVA and Chi-Square test. Multivariate logistic regression was used to evaluate the relationship between Cys $C$ and carotid plaque phenotype. Other covariates included age, sex, serum LDL and HDL (sls=0.05, els $=0.10)$. Finally, smoothing function curve and a three-piecewise linear regression were used to evaluate the relationship between serum Cys $\mathrm{C}$ and carotid plaque burden. All analyses were performed using SPSS 13.0.

\section{Results}

\section{Demographic characteristics}

Overall, patients with carotid plaque were more likely to be older age, have higher levels of serum Cys C, LDL, SBP, as well as higher prevalence of hypertension and diabetes, comparing to patients with no lesions. In addition, $n$ the group of patients without established renal dysfunction, the prevalence of smokingalcohol usewere higher among patients with carotid stable plaque than those with no lesions. While within the group of patients without established renal dysfunction, there was no significant difference in levels of serum Cys $C$ between patients with unstable plaque and patients with no lesions.The prevalence of physical exercise in patients with carotid plaque was lower than the patients with no lesions in different kidney function. The plaque burden between stable plaque and unstable plaque group has no significant difference. The detailed results were shown in Table 1.

\section{Influencing factors of carotid plaque phenotype}

Table 2 showed that Cys $\mathrm{C}$ was not only a risk factor for stable plaque, but also was a risk factor for unstable plaque in patients with mild decreased GFR.. However, among patients with normal GFR, serum Cys $\mathrm{C}$ was only a risk factor for stable plaque but not for unstable plaque (Figure 1, 2).

The correlation analysis between plaque burden and serum Cys $C$

Smoothing function curve analysis suggested a nonlinear relationship between Cys $C$ and plaque area and Crouse score adjusting for age, sex, serum LDL and HDL (Figure 1-2). A three-piecewise linear regression showed that the plaque score and plaque area tended to increase regardless of the status of renal function while serum Cys $\mathrm{C}$ floated in the range of $0.75-1.29(\mathrm{mg} / \mathrm{L})$, (Correction factors according to the results of multivariate logistic regression) (Table 3). 


\section{Kidney \\ Blood Pressure Research}

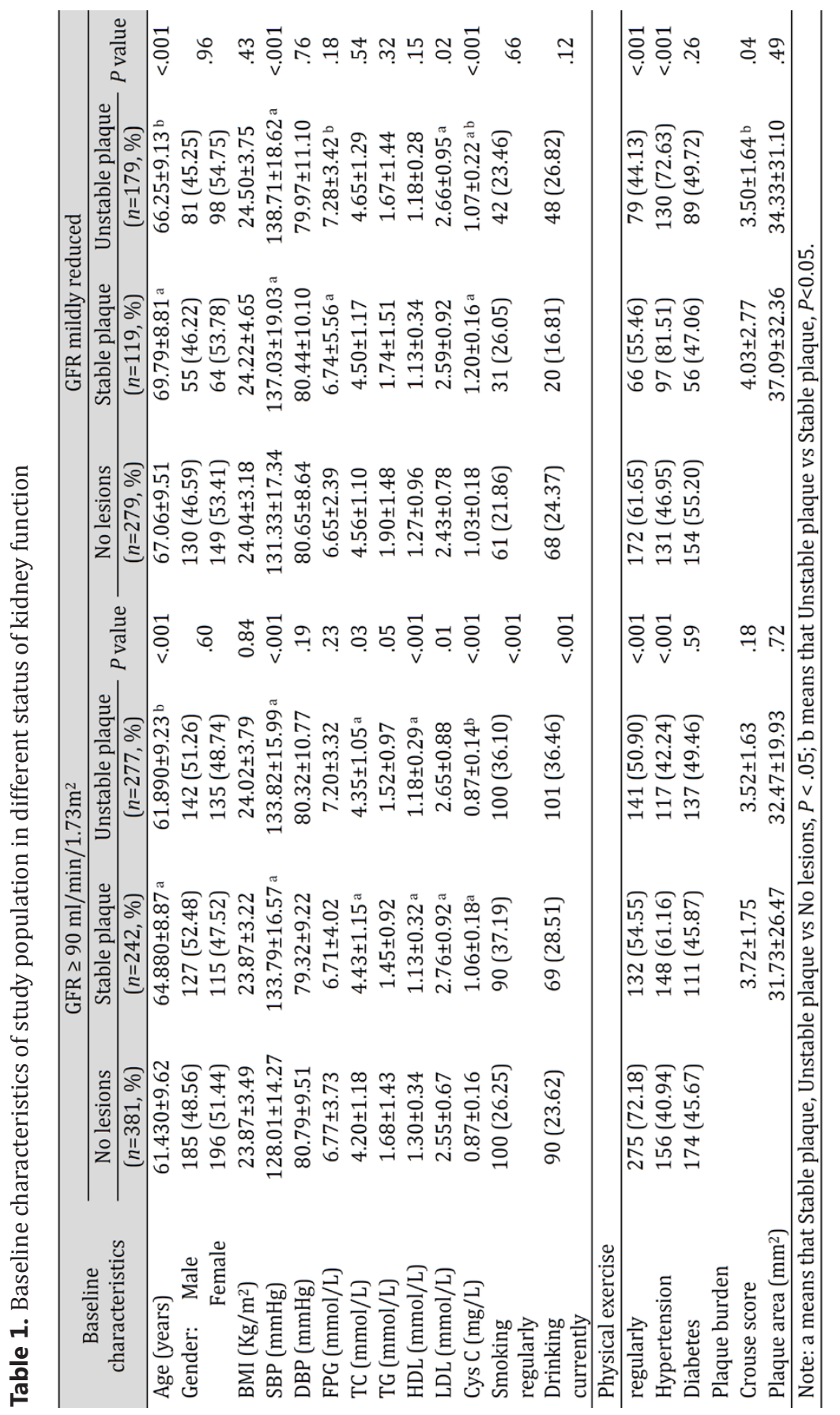

\section{Discussion}

In this study, our results suggested that, among patients with mild impaired renal function, Cys C levels were associated carotid artery lesions. In routine clinical practice, GFR valueis usually estimated based on the circulating concentration of an endogenous marker, such as creatinine, due to the infeasibility of direct measurement of actual GFR value. Cys $\mathrm{C}$ has emerged as a highly sensitive marker of even mildly impaired GFR [24, 25]. Indeed, because of its small size $(13 \mathrm{kDa})$, Cys-C is freely filtred by the glomerulus and neither secreted nor reabsorbed by tubular epithelial cells of kidneys. A reduced GFR is a strong independent predictor of all-cause and cardiovascular mortality, and is considered as a cardiovascular risk, as well as a indicator of previous history of myocardial infarcti-

on $[26,27]$. Detailed mechanism of the linkage between Cys $C$ and carotid artery lesions has not been fully elucidated. Renal mechanism seems to be a plausible link between increased Cys $\mathrm{C}$ and CVD. It has been widely recognized that cardiovascular events frequently occur in patients with chronic kidney disease (CKD) [28, 29]. Renin-angiotensin-aldosterone system (RAAS) plays a key role in the process of CKD. Serum Cys $C$ was assumed to be a sensitive indicator of "pre-clinical" renal disease which can not be detected by GFR based on serum $\mathrm{Cr}$ [30]. Serum Cys $C$ was identifies mild renal dysfunction. There is increased activation of the RAAS system with potentially deleterious effects. 


\section{Kidney Blood Pressure Research}

Table 2. The analysis of Cys $\mathrm{C}$ effect on plaque phenotype by multinomial logistic regression in different status of kidney function

\begin{tabular}{cccccccc}
\hline \multirow{2}{*}{ Impact Factor } & \multirow{2}{*}{ Plaque phenotype } & \multicolumn{3}{c}{ GFR $\geq 90 \mathrm{ml} / \mathrm{min} / 1.73 \mathrm{~m}^{2}$} & \multicolumn{2}{c}{ GFR mildly reduced } \\
\cline { 3 - 8 } & & $\beta$ & $O R(95 \% \mathrm{C})$ & $P$ value & $\beta$ & OR(95\%Cl) & $P$ value \\
\hline \multirow{2}{*}{ Cys C } & Stable plaque & 0.72 & $2.05(1.83-2.29)$ & $<.001$ & 0.47 & $1.60(1.43-1.78)$ & $<.001$ \\
& Unstable plaque & -0.03 & $0.97(0.88-1.06)$ & .49 & 0.19 & $1.21(1.10-1.32)$ & $<.001$ \\
\hline Correction factors & & & & & & & \\
\hline \multirow{2}{*}{ Age } & Stable plaque & 0.02 & $1.02(1.00-1.04)$ & .04 & 0.01 & $1.01(0.99-1.04)$ & .38 \\
& Unstable plaque & 0.01 & $1.01(0.99-1.03)$ & .09 & -0.03 & $0.98(0.96-0.99)$ & .01 \\
Hypertension & Stable plaque & 0.32 & $1.38(0.94-2.02)$ & .10 & 1.38 & $3.97(2.42-6.51)$ & $<.001$ \\
& Unstable plaque & -0.68 & $0.51(0.36-0.71)$ & $<.001$ & 0.88 & $2.41(1.65-3.52)$ & $<.001$ \\
Smoking: regularly & Stable plaque & -0.12 & $0.88(0.55-1.41)$ & .61 & 1.41 & $4.10(1.77-9.51)$ & $<.001$ \\
& Unstable plaque & -0.22 & $0.80(0.54-1.20)$ & .28 & -0.31 & $0.73(0.38-1.40)$ & .34 \\
Drinking: currently & Stable plaque & 0.04 & $1.04(0.64-1.71)$ & .86 & -1.92 & $0.15(0.06-0.35)$ & $<.001$ \\
Physical exercise: & Unstable plaque & 0.56 & $1.75(1.17-2.63)$ & $<.001$ & 0.12 & $1.13(0.61-2.09)$ & .70 \\
regularly & Stable plaque & -0.70 & $0.50(0.35-0.70)$ & $<.001$ & -0.39 & $0.68(0.45-1.02)$ & .060 \\
& Unstable plaque & -0.74 & $0.48(0.36-0.64)$ & $<.001$ & -0.99 & $0.37(0.27-0.52)$ & $<.001$ \\
TC & Stable plaque & 0.10 & $1.11(0.93-1.33)$ & .26 & -0.15 & $0.86(0.68-1.09)$ & .21 \\
& Unstable plaque & 0.10 & $1.11(0.95-1.29)$ & .18 & -0.13 & $0.88(0.73-1.04)$ & .13 \\
HDL & Stable plaque & -0.74 & $0.48(0.29-0.79)$ & $<.001$ & -0.37 & $0.69(0.41-1.16)$ & .16 \\
& Unstable plaque & -1.26 & $0.28(0.19-0.43)$ & $<.001$ & -0.23 & $0.79(0.61-1.04)$ & .08 \\
LDL & Stable plaque & -0.05 & $0.95(0.75-1.20)$ & .65 & 0.52 & $1.69(1.23-2.31)$ & $<.001$ \\
& Unstable plaque & 0.11 & $1.12(0.91-1.37)$ & .28 & 0.44 & $1.55(1.21-1.97)$ & $<.001$ \\
\hline
\end{tabular}
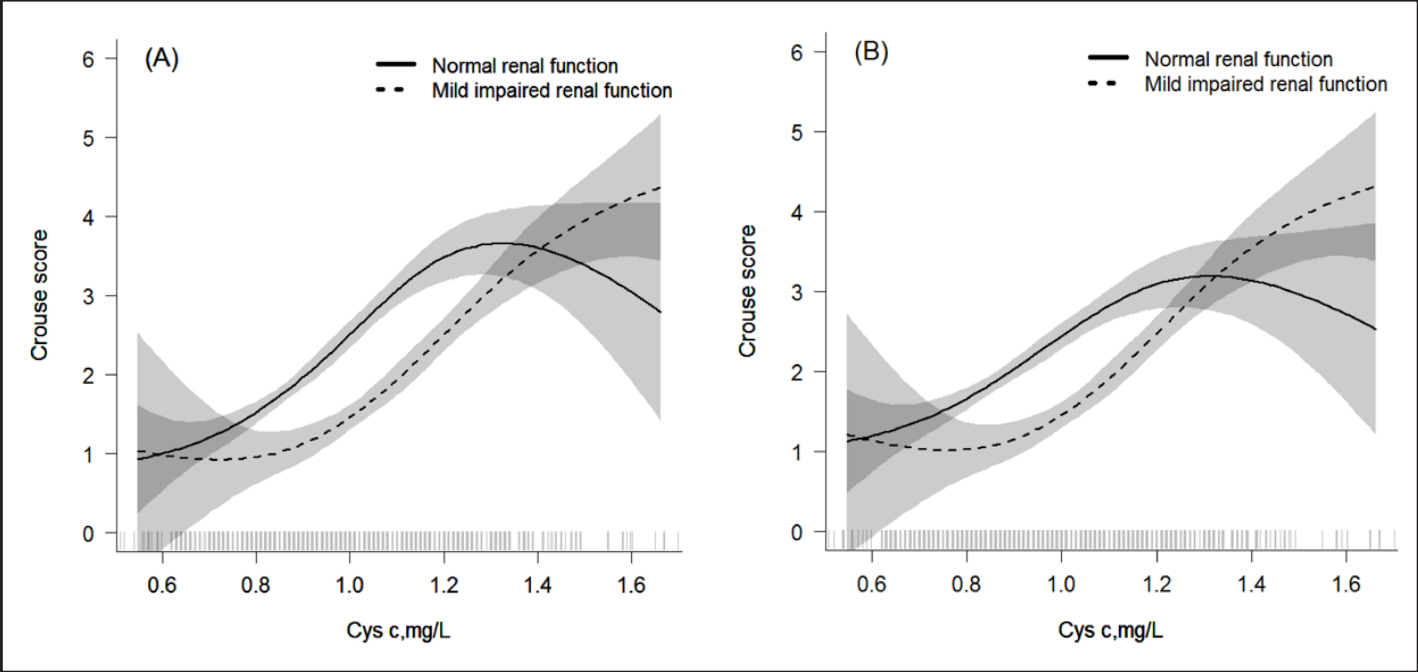

Fig. 1. The relationship between Cys C (mg/L) and Crouse score and their 95\% confidence intervals (shade scope) in patients with normal renal function (solid line, factor " 1 " = normal renal function) and in patients with mild impaired renal function (dashed line, factor " 2 " = mild impaired renal function), which using generalized smoothing splines with 5 knots. When serum cystatin C was in the range of $0.75-1.30$ (mg/L), the Crouse score tended to increase, and their $95 \%$ confidence intervals less volatile. A: no adjusted; B: adjusted for age, sex, serum LDL and HDL.

Interestingly, a few recent studies reported consistent findings showing that cardiovascular risk starts increasing for GFR still within the normal range [31]. In this study, we demonstrated that Cys $\mathrm{C}$ was a risk factor of stable plaque, and the increased level of serum Cys $\mathrm{C}$ may benefit to the stability of plaques in patients with normal renal function. Rupture of the cap of atherosclerotic plaques is considered to be a crucial step in the development of myocardial infarction and stroke $[32,33]$. Sudden alterations in atherosclerotic plaque structure such as cap rupture and intra plaque hemorrhage frequently precede ischemic 


\section{Kidney Blood Pressure Research}

Table 3. Threshold effect analysis of serum Cys C on plaque burden using linear regression

\begin{tabular}{|c|c|c|c|c|c|c|c|c|c|c|c|c|}
\hline \multirow{3}{*}{ plaque burden } & \multicolumn{6}{|c|}{$\mathrm{GFR} \geq 90 \mathrm{ml} / \mathrm{min} / 1.73 \mathrm{~m}^{2}$} & \multicolumn{6}{|c|}{ GFR mildly reduced } \\
\hline & \multicolumn{3}{|c|}{ Crude } & \multicolumn{3}{|c|}{ Adjusted } & \multicolumn{3}{|c|}{ Crude } & \multicolumn{3}{|c|}{ Adjusted } \\
\hline & $\beta$ & S.E & Pvalue & $\beta$ & S.E & Pvalue & $\beta$ & S.E & Pvalue & $\beta$ & S.E & Pvalue \\
\hline \multicolumn{13}{|l|}{ Crouse score } \\
\hline Cys C: $<0.75(\mathrm{mg} / \mathrm{L})$ & 4.40 & 3.08 & .16 & 3.79 & 3.14 & 23 & -5.39 & 9.04 & 0.79 & -2.00 & 2.15 & .50 \\
\hline $0.75 \sim$ & 1.98 & 0.55 & $<.001$ & 1.34 & 0.61 & .03 & 2.75 & 0.87 & $<0.01$ & 2.69 & 0.87 & $<.001$ \\
\hline $1.30 \sim$ & -6.12 & 17.68 & .89 & -5.04 & 14.75 & .76 & 0.66 & 2.77 & 0.81 & 0.46 & 3.38 & .89 \\
\hline \multicolumn{13}{|l|}{ Plaque area } \\
\hline Cys C: $<0.75(\mathrm{mg} / \mathrm{L})$ & 33.59 & 27.52 & .22 & 44.14 & 28.40 & .13 & 6.90 & 5.53 & 0.27 & 5.72 & 3.48 & .11 \\
\hline $0.75 \sim$ & 28.86 & 7.15 & $<.001$ & 16.39 & 7.64 & .03 & 39.40 & 11.65 & $<0.01$ & 38.77 & 11.39 & $<.001$ \\
\hline $1.30 \sim$ & -94.78 & 158.90 & .57 & -230.71 & 418.77 & .62 & -37.12 & 49.08 & 0.49 & -54.19 & 53.27 & .31 \\
\hline
\end{tabular}

Crude: unadjusted. Adjusted for age, sex, serum LDL and HDL

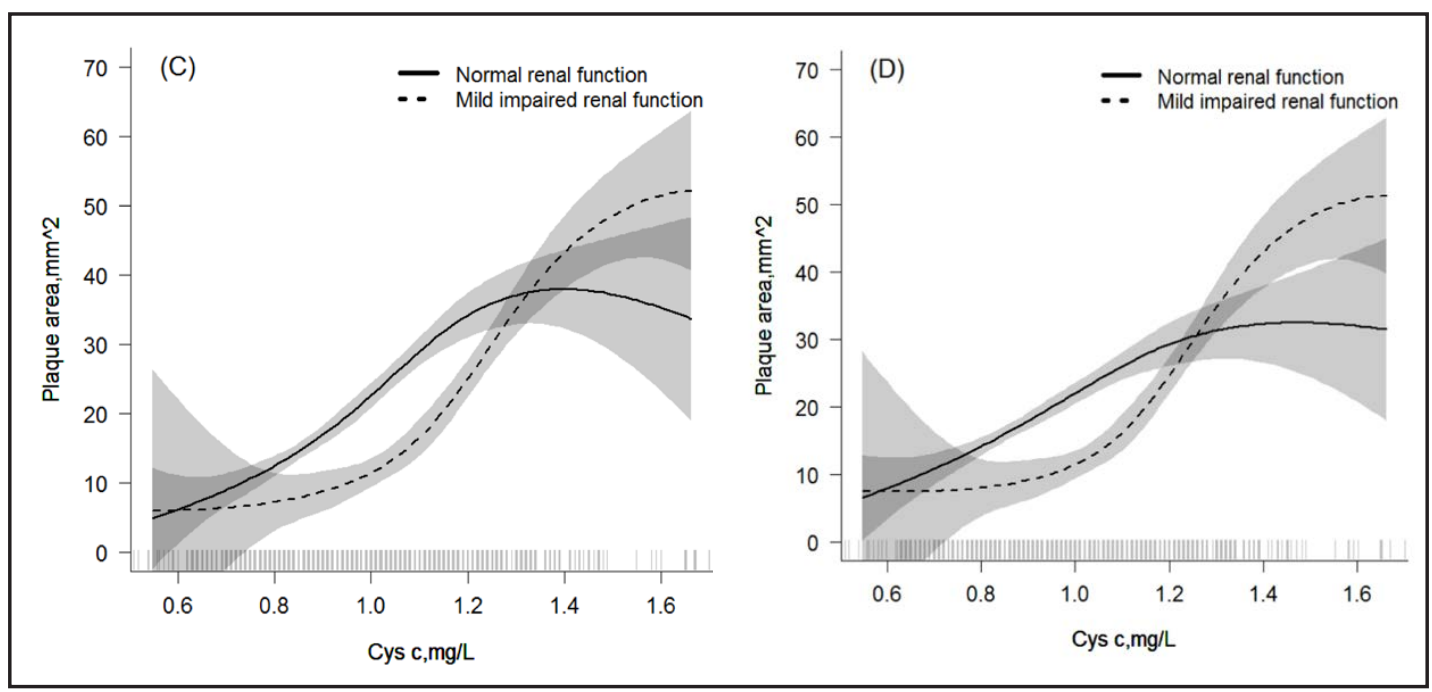

Fig. 2. The relationship between Cys C (mg/L) and plaque area $\left(\mathrm{mm}^{2}\right)$ and their $95 \%$ confidence intervals (shade scope) in patients with normal renal function (solid line, factor "1"= normal renal function) and in patients with mild impaired renal function (dashed line, factor " 2 "= mild impaired renal function), which using generalized smoothing splines with 5 knots. When serum cystatin $C$ was in the range of $0.75-1.30$ (mg/L), the plaque area tended to increase, and their 95\% confidence intervals less volatile. C: no adjusted; D: adjusted for age, sex, serum LDL and HDL.

cardiovascular events. It has been suggested that plaque rupture is caused by a localized imbalance in proteolytic activity, and of particular interest is the family of enzymes known as the matrix metalloproteinases and cathepsin $[34,35]$. The cap of the plaque is composed predominantly of type III collagen [36]. Collagen is synthesized by smooth muscle cells (SMCs). Thus, increased collagen and elastic fibers could result from an increased number of SMCs or increased extracellular matrix synthesis by these cells. Cys $\mathrm{C}$ is a member of the cystatin superfamily of endogenous cysteine protease inhibitors that inhibits the protease of cathepsin and which is produced by all nucleated cells. The dynamic unbalance of cathepsin and its inhibitor-Cys $\mathrm{C}$ was the main reason leading to extracellular matrix remodeling. However, the dynamic balance of formation and degradation in ECM plays a key role in the development and progression of plaque phenotype. Normal physiological remodeling allows the adult blood vessel to adapt and repair, whereas perturbation of vascular remodeling is an important component of the pathogenesis of atherosclerosis, plaque rupture, restenosis, and aneurysm formation. Overexpression of matrix metalloproteinase and cysteine protease, known as cathepsins, also has been implicated in atherosclerosis, and the potential 


\section{Kidney \\ Blood Pressure Research}

Kidney Blood Press Res 2016;41:197-207

\begin{tabular}{l|l}
\hline DOI: 10.1159/000443422 & ( 2016 The Author(s). Published by S. Karger AG, Basel
\end{tabular}

Published online: March 25, 2016

www.karger.com $/ \mathrm{kb}$

204

significance of imbalance between cysteine proteases and their most abundant inhibitor-Cys $\mathrm{C}$ has been highlighted by the demonstration of Cys $\mathrm{C}$ deficiency in acute myocardial infarction [37]. Previous studies have revealed that the severe depression of cystatin $C$ antigen levels within SMCs of atherosclerotic plaques and which implied a marked change in the functional properties of plaque SMC [38]. Deo R et al. have also revealed that the elevated levels of Cys $\mathrm{C}$ were associated with inducible ischemia among outpatients with stable coronary disease [39].

Additionally, in this study we also demonstrate that a nonlinear relationship was observed between serum Cys $\mathrm{C}$ and plaque burden after adjusted for age, sex, serum LDL and HDL. When serum Cys $\mathrm{C}$ was in the range of 0.75-1.30 (mg/L), the plaque burden tended to increase. In an early state of CAS, LDL accumulates in the intimal layer in blood vessels. Oxidation of the LDL induces recruitment of inflammatory cells, such as bone marrowderived monocytes and T lymphocytes [40]. Later, SMCs from the media migrate through the internal elastic laminae, and abnormal proliferation. The abnormal proliferation of SMCs expresses abundant collagen, especially in the development and progression of CVD, and forms a thickly and elastic fibrous cap over a core of lipids, inflammatory and necrotic cells [40]. Cys $C$ is a member of the cystatin superfamily of endogenous cysteine protease inhibitor and which can inhibit the degradation of ECM [41]. The ECM of the vessel wall is composed of collagen, elastin, proteoglycans and a number of other glycoproteins. Both elastin and collagen are important structural proteins, forming plaque fibrous cap and giving elastic and tensile properties to ECM [42]. Gu F et al. have revealed that serum cystatin C positively correlated with plaque area $(r=0.467, P<.001)$ and plaque burden $(r=0.395$, $P=.003$ ) in patients with unstable angina [43]. Silva D et al. also found that a high baseline Cys $C$ level was associated with an increased risk of death $(O R=8.50 ; P<.001)$ and death or reinfarction $(O R=3.89 ; P=.02)$ [44]. Our findings are also in line with those by Niccoli et al, who found that cystatin $C$ independently predicted coronary atherosclerotic burden and long-term prognosis [45].

This study has several limitations. First, this was a human-based study, we have found a phenomenon that a nonlinear relationship was observed between serum Cys C and plaque burden and the serum Cys $C$ was in the range of $0.75-1.30(\mathrm{mg} / \mathrm{L})$, the plaque burden tended to increase. However, animal experiments were not carried out to explore the detailed mechanisms. In the future study, we will establish animal model to explore the causal relationship of them. Secondly, we used MDRD formula to estimate the renal function. The accuracy of renal function evaluation could be improved by applying actual GFR measurements in future studies.

\section{Conclusion}

Serum Cys C may benefit to the stability of plaques among patients with normal kidney function. Within a certain range, serum Cys $C$ is associated with an increased carotid plaque burden.

\section{Disclosures}

The authors of this manuscript state that they do not have any conflict of interests and nothing to disclose. 


\section{Kidney \\ Blood Pressure Research}

\section{Acknowledgments}

First of all, we would like to thank all the staff from the Department of Medical Ultrasonics, Ma'anshan People's Hospital for technical assistance. Secondly we wish to thank the participants for their co-operation in data collection.

\section{References}

1 Corrales A, Parra JA, González-Juanatey C, Rueda-Gotor J, Blanco R, Llorca J, González-Gay MA:

Cardiovascular risk stratification in rheumatic diseases: carotid ultrasound is more sensitive than Coronary Artery Calcification Score to detect subclinical atherosclerosis in patients with rheumatoid arthritis. Ann Rheum Dis 2013;72:1764-1770.

2 Zavodni AE, Wasserman BA, McClelland RL, Gomes AS, Folsom AR, Polak JF, Lima JA, Bluemke DA: Carotid artery plaque morphology and composition in relation to incident cardiovascular events: the Multi-Ethnic Study of Atherosclerosis (MESA). Radiology 2014;271:381-389.

3 Sillesen H, Muntendam P, Adourian A, Entrekin R, Garcia M, Falk E, Fuster V: Carotid plaque burden as a measure of subclinical atherosclerosis: comparison with other tests for subclinical arterial disease in the High Risk Plaque BioImage study. JACC Cardiovasc Imaging 2012;5:681-689.

4 Corban MT, Eshtehardi P, Suo J, McDaniel MC, Timmins LH, Rassoul-Arzrumly E, Maynard C, Mekonnen G, King S, Quyyumi AA: Combination of plaque burden, wall shear stress, and plaque phenotype has incremental value for prediction of coronary atherosclerotic plaque progression and vulnerability. Atherosclerosis 2014;232:271-276.

5 Störk S, van den Beld AW, von Schacky C, Angermann CE, Lamberts SW, Grobbee DE, Bots ML: Carotid artery plaque burden, stiffness, and mortality risk in elderly men a prospective, population-based cohort study. Circulation 2004;110:344-348.

6 Homburg PJ, Rozie S, van Gils MJ, Jansen T, de Weert TT, Dippel DW, van der Lugt A: Atherosclerotic plaque ulceration in the symptomatic internal carotid artery is associated with nonlacunar ischemic stroke. Stroke 2010;41:1151-1156.

7 Baumgartner R: Biglycan: Unpuzzling the causal links between tobacco-smoking and atherosclerosis? Atherosclerosis 2014;237:809-810.

8 Jung J-M, Young Kwon D, Han C, Park MH: Metabolic syndrome and early carotid atherosclerosis in the elderly. J Atheroscler Thromb 2014;21:435-444.

9 Novo S, Peritore A, Trovato RL, Guarneri FP, Di Lisi D, Muratori I, Novo G: Preclinical atherosclerosis and metabolic syndrome increase cardio-and cerebrovascular events rate: a 20-year follow up. Cardiovasc Diabetol 2013;12:155.

10 Gómez-Marcos MA, Recio-Rodríguez JI, Rodríguez-Sánchez E, Castaño-Sánchez Y, de Cabo-Laso A, SánchezSalgado B, Rodríguez-Martín C, Castaño-Sánchez C, Gómez-Sánchez L, García-Ortiz L: Central blood pressure and pulse wave velocity: relationship to target organ damage and cardiovascular morbiditymortality in diabetic patients or metabolic syndrome. An observational prospective study. LOD-DIABETES study protocol. BMC public health 2010;10:143.

11 Obunai K, Jani S, Dangas GD: Cardiovascular morbidity and mortality of the metabolic syndrome. Med Clin North Am 2007;91:1169-1184.

12 Luc G, Bard J-M, Lesueur C, Arveiler D, Evans A, Amouyel P, Ferrieres J, Juhan-Vague I, Fruchart J-C, Ducimetiere P: Plasma cystatin-C and development of coronary heart disease: The PRIME Study. Atherosclerosis 2006;185:375-380.

13 Grams ME, Juraschek SP, Selvin E, Foster MC, Inker LA, Eckfeldt JH, Levey AS, Coresh J: Trends in the Prevalence of Reduced GFR in the United States: A Comparison of Creatinine-and Cystatin C- Based Estimates. Am J Kidney Dis 2013;62:253-260.

14 Arun O, Celik G, Oc B, Unlu A, Celik JB, Oc M, Duman A: Renal Effects of Coronary Artery Bypass Graft Surgery in Diabetic and Non-Diabetic Patients: A Study with Urinary Neutrophil Gelatinase-Associated Lipocalin and Serum Cystatin C. Kidney Blood Press Res 2015;40:141-152. 


\section{Kidney \\ Blood Pressure Research}

15 Zhu Y, Zhang H-P, Wang Y-C, Ren T-T, Li J, Xu M-L, Wang X-Q, Liu F-C, Lau A, Wen Y-F: Serum cystatin C level is associated with carotid intima-media thickening and plaque. Scand J Clin Lab Invest 2015;75:265-272.

16 Zhang H, Liu M, Ren T, Wang X, Liu D, Xu M, Han L, Wu Z, Li H, Zhu Y: Associations between Carotid Artery Plaque Score, Carotid Hemodynamics and Coronary Heart Disease. Int J Environ Res Public Health 2015;12:14275-14284.

17 Romero JR, Vasan RS, Beiser AS, Polak JF, Benjamin EJ, Wolf PA, Seshadri S: Association of carotid artery atherosclerosis with circulating biomarkers of extracellular matrix remodeling: the Framingham Offspring Study. J Stroke Cerebrovasc Dis 2008;17:412-417.

18 Xie L, Terrand J, Xu B, Tsaprailis G, Boyer J, Chen QM: Cystatin C increases in cardiac injury: a role in extracellular matrix protein modulation. Cardiovasc Res 2010;87:628-635.

19 Muntner P, Mann D, Winston J, Bansilal S, Farkouh ME: Serum cystatin C and increased coronary heart disease prevalence in US adults without chronic kidney disease. Am J Cardiol 2008;102:54-57.

20 Doganer YC, Aydogan U, Aydogdu A, Aparci M, Akbulut H, Nerkiz P, Turker T, Cayci T, Barcin C, Saglam K: Relationship of cystatin C with coronary artery disease and its severity. Coron Artery Dis 2013;24:119-126.

21 Levey AS, Bosch JP, Lewis JB, Greene T, Rogers N, Roth D: A more accurate method to estimate glomerular filtration rate from serum creatinine: a new prediction equation. Ann Intern Med 1999;130:461-470.

22 Urbano LA, Fabienne P, Rossetti AO, Ludwig VS, Julien B, Gérald D: Images in cardiovascular medicine. Thrombus in the internal carotid artery complicating an "unstable" atheromatous plaque. Circulation 2003;107:e19-20.

23 Kawai T, Ohishi M, Takeya Y, Onishi M, Ito N, Oguro R, Yamamoto K, Kamide K, Rakugi H: Carotid plaque score and intima media thickness as predictors of stroke and mortality in hypertensive patients. Hypertens Res 2013;36:902-909.

24 Stevens LA, Coresh J, Greene T, Levey AS: Assessing kidney function - measured and estimated glomerular filtration rate. N Engl J Med 2006;354:2473-2483.

25 Luis D, Huang X, Riserus U, Sjögren P, Lindholm B, Arnlöv J, Cederholm T, Carrero JJ: Estimated dietary acid load is not associated with blood pressure or hypertension incidence in men who are approximately 70 years old. J Nutr 2015;145:315-321.

26 van der Velde M, Matsushita K, Coresh J, Astor BC, Woodward M, Levey A, de Jong P, Gansevoort RT, Levey AS, de Jong PE: Lower estimated glomerular filtration rate and higher albuminuria are associated with allcause and cardiovascular mortality. A collaborative meta-analysis of high-risk population cohorts. Kidney Int 2011;79:1341-1352.

27 Tonelli M, Muntner P, Lloyd A, Manns BJ, Klarenbach S, Pannu N, James MT, Hemmelgarn BR, Network AKD: Risk of coronary events in people with chronic kidney disease compared with those with diabetes: a population-level cohort study. Lancet 2012;380:807-814.

28 Ekart R, Bevc S, Hojs N, Knehtl M, Dvoršak B, Hojs R: Albuminuria is Associated With Subendocardial Viability Ratio in Chronic Kidney Disease Patients. Kidney Blood Press Res 2015;40:565-574.

29 Anavekar NS, McMurray JJ, Velazquez EJ, Solomon SD, Kober L, Rouleau J-L, White HD, Nordlander R, Maggioni A, Dickstein K: Relation between renal dysfunction and cardiovascular outcomes after myocardial infarction. N Engl J Med 2004;351:1285-1295.

30 McMurray MD, Trivax JE, McCullough PA: Serum cystatin C, renal filtration function, and left ventricular remodeling. Circ Heart Fail 2009;2:86-89.

31 Levin A: Cystatin C, serum creatinine, and estimates of kidney function: searching for better measures of kidney function and cardiovascular risk. Ann Inter Med 2005;142:586-588.

32 Falk E, Shah PK, Fuster V: Coronary plaque disruption. Circulation 1995;92:657-671.

33 Shah PK: Plaque disruption and thrombosis: potential role of inflammation and infection. Cardiol Rev 2000;8:31-39.

34 Li W, Kornmark L, Jonasson L, Forssell C, Yuan X-M: Cathepsin L is significantly associated with apoptosis and plaque destabilization in human atherosclerosis. Atherosclerosis 2009;202:92-102.

35 Higashikata T, Yamagishi M, Higashi T, Nagata I, Iihara K, Miyamoto S, Ishibashi-Ueda H, Nagaya N, Iwase T, Tomoike H: Altered expression balance of matrix metalloproteinases and their inhibitors in human carotid plaque disruption: results of quantitative tissue analysis using real-time RT-PCR method. Atherosclerosis 2006;185:165-172. 


\section{Kidney \\ Blood Pressure Research}

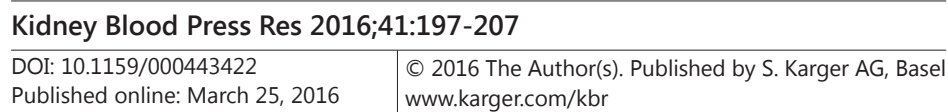

Wen/Xia/Wang/Zhang/Li/Ali/Gao/Li/Sun/Li: A Clinic Based Study

36 Lopes J, Adiguzel E, Gu S, Liu S-L, Hou G, Heximer S, Assoian RK, Bendeck MP: Type VIII collagen mediates vessel wall remodeling after arterial injury and fibrous cap formation in atherosclerosis. Am J Pathol 2013;182:2241-2253.

37 Noto D, Cefalu AB, Barbagallo CM, Pace A, Rizzo M, Marino G, Caldarella R, Castello A, Pernice V, Notarbartolo A: Cystatin C levels are decreased in acute myocardial infarction: effect of cystatin C G73A gene polymorphism on plasma levels. Int J Cardiol 2005;101:213-217.

38 Shi G-P, Sukhova GK, Grubb A, Ducharme A, Rhode LH, Lee RT, Ridker PM, Libby P, Chapman HA: Cystatin C deficiency in human atherosclerosis and aortic aneurysms. J Clin Invest 1999;104:1191-1197.

39 Deo R, Shlipak MG, Ix JH, Ali S, Schiller NB, Whooley MA: Association of cystatin C with ischemia in patients with coronary heart disease. Clin Cardiol 2009;32:E18-E22.

40 Epstein FH, Ross R: Atherosclerosis - an inflammatory disease. N Engl J Med 1999;340:115-126.

41 Papafaklis MI, Koskinas KC, Chatzizisis YS, Stone PH, Feldman CL: In-vivo assessment of the natural history of coronary atherosclerosis: vascular remodeling and endothelial shear stress determine the complexity of atherosclerotic disease progression. Curr Opin Cardiol 2010;25:627-638.

42 Bengtsson E, To F, Grubb A, Håkansson K, Wittgren L, Nilsson J, Jovinge S: Absence of the protease inhibitor cystatin $\mathrm{C}$ in inflammatory cells results in larger plaque area in plaque regression of apoE-deficient mice. Atherosclerosis 2005;180:45-53.

43 Gu F, Lü S, Chen Y, Zhou Y, Song X, Jin Z, Liu H: Relationship between plasma cathepsin S and cystatin C levels and coronary plaque morphology of mild to moderate lesions: an in vivo study using intravascular ultrasound. Chin Med J (Engl) 2009;122:2820-2826.

44 Silva D, Cortez-Dias N, Jorge C, Marques JS, Carrilho-Ferreira P, Magalhães A, Martins SR, Gonçalves S, da Silva PC, Fiúza M: Cystatin C as prognostic biomarker in ST-segment elevation acute myocardial infarction. Am J Cardiol 2012;109:1431-1438.

45 Niccoli G, Conte M, Della Bona R, Altamura L, Siviglia M, Dato I, Ferrante G, Leone AM, Porto I, Burzotta F: Cystatin $C$ is associated with an increased coronary atherosclerotic burden and a stable plaque phenotype in patients with ischemic heart disease and normal glomerular filtration rate. Atherosclerosis 2008;198:373-380. 